\title{
Fast Perceptron Decision Tree Learning from Evolving Data Streams
}

\author{
Albert Bifet, Geoff Holmes, Bernhard Pfahringer, and Eibe Frank \\ University of Waikato, Hamilton, New Zealand \\ \{abifet, geoff, bernhard, eibe\}@cs. waikato.ac.nz
}

\begin{abstract}
Mining of data streams must balance three evaluation dimensions: accuracy, time and memory. Excellent accuracy on data streams has been obtained with Naive Bayes Hoeffding Trees-Hoeffding Trees with naive Bayes models at the leaf nodes - albeit with increased runtime compared to standard Hoeffding Trees. In this paper, we show that runtime can be reduced by replacing naive Bayes with perceptron classifiers, while maintaining highly competitive accuracy. We also show that accuracy can be increased even further by combining majority vote, naive Bayes, and perceptrons. We evaluate four perceptron-based learning strategies and compare them against appropriate baselines: simple perceptrons, Perceptron Hoeffding Trees, hybrid Naive Bayes Perceptron Trees, and bagged versions thereof. We implement a perceptron that uses the sigmoid activation function instead of the threshold activation function and optimizes the squared error, with one perceptron per class value. We test our methods by performing an evaluation study on synthetic and real-world datasets comprising up to ten million examples.
\end{abstract}

\section{Introduction}

In the data stream model, data arrive at high speed, and algorithms that process them must do so under very strict constraints of space and time. Consequently, data streams pose several challenges for data mining algorithm design. First, algorithms must make use of limited resources (time and memory). Second, they must deal with data whose nature or distribution changes over time.

An important issue in data stream mining is the cost of performing the learning and prediction process. As an example, it is possible to buy time and space usage from cloud computing providers [25]. Several rental cost options exist:

- Cost per hour of usage: Amazon Elastic Compute Cloud (Amazon EC2) is a web service that provides resizable compute capacity in the cloud. Cost depends on the time and on the machine rented (small instance with $1.7 \mathrm{~GB}$, large with $7.5 \mathrm{~GB}$ or extra large with $15 \mathrm{~GB}$ ).

- Cost per hour and memory used: GoGrid is a web service similar to Amazon EC2, but it charges by RAM-Hours. Every GB of RAM deployed for 1 hour equals one RAM-Hour. 
It is crucial to find mining methods that use resources efficiently. In this spirit, we propose in this paper the Hoeffding Perceptron Tree for classification, as a faster method compared to the state-of-the-art Hoeffding Tree with naive Bayes leaves. The idea is to implement perceptron classifiers at the leaves of the Hoeffding Tree, to potentially increase accuracy, but mainly to reduce runtime.

We introduce the use of RAM-Hours as an evaluation measure of the resources used by streaming algorithms. The paper is structured as follows: related work is presented in Section 2. Hoeffding Perceptron Trees and bagging of such trees are discussed in Section 3. An experimental evaluation is conducted in Section 4. Finally, conclusions and suggested items for future work are presented in Section 5 .

\section{Related Work}

Standard decision tree learners such as ID3, C4.5, and CART $[18,21]$ assume that all training examples can be stored simultaneously in main memory, and are thus severely limited in the number of examples they can learn from. In particular, they are not applicable to data streams, where potentially there is no bound on the number of examples and these arrive sequentially.

Domingos et al. $[6,14]$ proposed the Hoeffding tree as an incremental, anytime decision tree induction algorithm that is capable of learning from data streams, assuming that the distribution generating examples does not change over time.

Hoeffding trees exploit the fact that a small sample can often suffice to choose a splitting attribute. This idea is supported by the Hoeffding bound, which quantifies the number of observations (in our case, examples) needed to estimate some statistics within a prescribed precision (in our case, the goodness of an attribute). More precisely, the Hoeffding bound states that with probability $1-\delta$, the true mean of a random variable of range $R$ will not differ from the estimated mean after $n$ independent observations by more than:

$$
\epsilon=\sqrt{\frac{R^{2} \ln (1 / \delta)}{2 n} .}
$$

A theoretically appealing feature of Hoeffding Trees not shared by other incremental decision tree learners (ID4 [22], ID5 [24]) is that it has sound guarantees of performance. Using the Hoeffding bound one can show that its output is asymptotically nearly identical to that of a non-incremental learner using infinitely many examples. CVFDT [14] is an extension of the Hoeffding Tree to evolving data streams, but does not exhibit theoretical guarantees.

Outside the data stream world, there is prior work on using perceptrons or similar classifiers in decision trees. Utgoff [24] presented the Perceptron Decision Tree as a decision tree in which each leaf node uses the perceptron as a classifier of the input instances. Bennett et al. [2] showed that maximizing margins in perceptron decision trees can be useful to combat overfitting. Zhou [26] proposed Hybrid Decision Trees as a hybrid learning approach combining decision trees with neural networks. 
Frank et al. [7] investigated using Model Trees for classification problems. Model trees are decision trees with linear regression functions at the leaves. Logistic Model Trees [17] are model trees that use logistic regression instead of linear regression. They have been shown to be very accurate and compact classifiers, but their induction is very time-consuming.

The LTree algorithm of Gama [8] embodies a general framework for learning functional trees, multivariate classification or regression trees that can use combinations of attributes at decision nodes, leaf nodes, or both.

In the data streams literature, Ikonomovska et al. [15] presented FIMT, a fast incremental model tree for regression on static data streams. To deal with concept drift, Ikonomovska et al. [16] proposed FIRT-DD as an adaption of the FIMT algorithm to time-changing distributions. FIMT and FIRT-DD use a perceptron learner at the leaves to perform regression. Considering classification methods for data streams, Bifet et al. [4] presented two new ensemble learning methods: one using bagging with decision trees of different size and one using ADWIN, an adaptive sliding window method that detects change and adjusts the size of the window correspondingly. We revisit the latter approach in this paper.

\section{Perceptrons and Hoeffding Perceptron Trees}

In this section, we present the perceptron learner we use, and the Hoeffding Perceptron Tree based on it. We also consider bagging trees with change detection.

\subsection{Perceptron Learning}

We use an online version of the perceptron that employs the sigmoid activation function instead of the threshold activation function and optimizes the squared error, with one perceptron per class value.

Given a data stream $\left\langle\boldsymbol{x}_{i}, y_{i}\right\rangle$, where $\boldsymbol{x}_{i}$ is an example and $y_{i}$ is its example class, the classifier's goal is to minimize the number of misclassified examples. Let $h_{\boldsymbol{w}}\left(\boldsymbol{x}_{i}\right)$ be the hypothesis function of the perceptron for instance $\boldsymbol{x}_{i}$. We use the mean-square error $J(\boldsymbol{w})=\frac{1}{2} \sum\left(y_{i}-h_{\boldsymbol{w}}\left(\boldsymbol{x}_{i}\right)\right)^{2}$ instead of the $0-1$ loss function, since it is differentiable.

The classic perceptron takes a linear combination and thresholds it. The predicted class of the perceptron is $h_{\boldsymbol{w}}\left(\boldsymbol{x}_{i}\right)=\operatorname{sgn}\left(\boldsymbol{w}^{T} \boldsymbol{x}_{i}\right)$, where a bias weight with constant input is included. Our hypothesis function $h_{\boldsymbol{w}}=\sigma\left(\boldsymbol{w}^{T} \boldsymbol{x}\right)$ instead uses the sigmoid function $\sigma(x)=1 /\left(1+e^{-x}\right)$ since it has the property $\sigma^{\prime}(x)=$ $\sigma(x)(1-\sigma(x))$. Thus, we can compute the gradient of the error function

$$
\nabla J=-\sum_{i}\left(y_{i}-h_{\boldsymbol{w}}\left(\boldsymbol{x}_{i}\right)\right) \nabla h_{\boldsymbol{w}}\left(\boldsymbol{x}_{i}\right)
$$

where for sigmoid hypothesis

$$
\nabla h_{\boldsymbol{w}}\left(\boldsymbol{x}_{i}\right)=h_{\boldsymbol{w}}\left(\boldsymbol{x}_{i}\right)\left(1-h_{\boldsymbol{w}}\left(\boldsymbol{x}_{i}\right)\right)
$$


obtaining the following weight update rule

$$
\boldsymbol{w}=\boldsymbol{w}+\eta \sum_{i}\left(y_{i}-h_{\boldsymbol{w}}\left(\boldsymbol{x}_{i}\right)\right) h_{\boldsymbol{w}}\left(\boldsymbol{x}_{i}\right)\left(1-h_{\boldsymbol{w}}\left(\boldsymbol{x}_{i}\right)\right) \boldsymbol{x}_{i}
$$

Because we work in a data stream scenario, rather than performing batch updates, we use stochastic gradient descent where the weight vector is updated after every example. As we deal with multi-class problems, we train one perceptron for each class. To classify an unseen instance $\boldsymbol{x}$, we obtain the predictions $h_{\boldsymbol{w}_{1}}(\boldsymbol{x}), \ldots, h_{\boldsymbol{w}_{n}}(\boldsymbol{x})$ from the perceptrons, and the predicted class is $\arg \max _{\text {class }} h_{\boldsymbol{w}_{\text {class }}}(\boldsymbol{x})$. The pseudocode is shown in Figure 1.

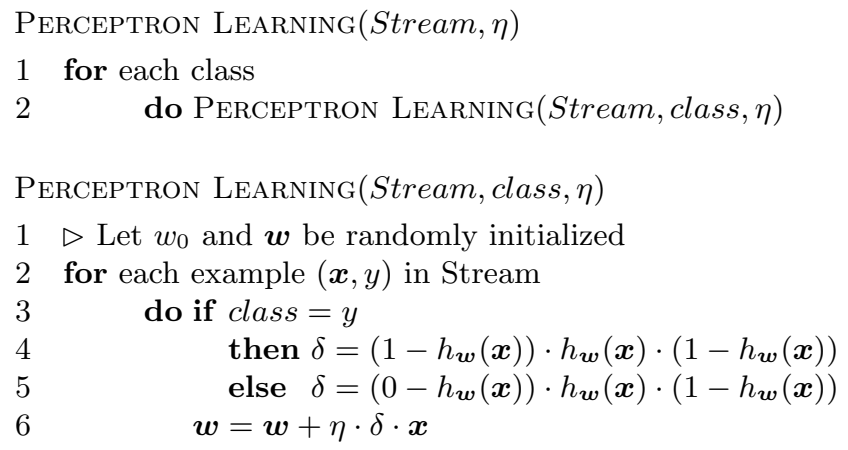

Perceptron Prediction $(\boldsymbol{x})$

1 return arg $\max _{\text {class }} h_{\boldsymbol{w}_{\text {class }}}(\boldsymbol{x})$

Fig. 1. Perceptron algorithm

\subsection{Hoeffding Perceptron Tree}

Hoeffding trees [6] are state-of-the-art in classification for data streams and they perform prediction by choosing the majority class at each leaf. Their predictive accuracy can be increased by adding naive Bayes models at the leaves of the trees. However, Holmes et al. [12] identified situations where the naive Bayes method outperforms the standard Hoeffding tree initially but is eventually overtaken. They propose a hybrid adaptive method that generally outperforms the two original prediction methods for both simple and complex concepts. We call this method Hoeffding Naive Bayes Tree (hnbt). This method works by performing a naive Bayes prediction per training instance, and comparing its prediction with the majority class. Counts are stored to measure how many times the naive Bayes prediction gets the true class correct as compared to the majority class. 
When performing a prediction on a test instance, the leaf will only return a naive Bayes prediction if it has been more accurate overall than the majority class, otherwise it resorts to a majority class prediction.

We adapt this methodology to deal with perceptrons rather than naive Bayes models. A Hoeffding Perceptron Tree (hpt) is a Hoeffding Tree that has a perceptron at each leaf. Similarly to hnbt, predictions by the perceptron are only used if they are more accurate on average than the majority class. It improves on hnbt in terms of runtime because it does not need to estimate the statistical distribution for numeric attributes and calculate density values based on the exponential function, and for discrete attributes it does not need to calculate divisions to estimate probabilities.

Finally, a Hoeffding Naive Bayes Perceptron Tree (hnbpt) is a Hoeffding Tree that has three classifiers at each leaf: a majority class, naive Bayes, and a perceptron. Voting is used for prediction. It is slower than the Hoeffding Perceptron Tree and the Hoeffding Naive Bayes Tree, but it combines the predictive power of the base learners.

\subsection{Bagging Trees with ADWIN}

ADWIN [3] is a change detector and estimator that solves in a well-specified way the problem of tracking the average of a stream of bits or real-valued numbers. ADWIN keeps a variable-length window of recently seen items, with the property that the window has the maximal length statistically consistent with the hypothesis "there has been no change in the average value inside the window".

ADWIN is parameter- and assumption-free in the sense that it automatically detects and adapts to the current rate of change. Its only parameter is a confidence bound $\delta$, indicating how confident we want to be in the algorithm's output, inherent to all algorithms dealing with random processes.

Also important for our purposes, ADWIN does not maintain the window explicitly, but compresses it using a variant of the exponential histogram technique. This means that it keeps a window of length $W$ using only $O(\log W)$ memory and $O(\log W)$ processing time per item.

ADWIN Bagging is the online bagging method of Oza and Russell [19] with the addition of the ADWIN algorithm as a change detector. When a change is detected, the worst classifier of the ensemble of classifiers is removed and a new classifier is added to the ensemble.

\section{Comparative Experimental Evaluation}

Massive Online Analysis (MOA) [13] is a software environment for implementing algorithms and running experiments for online learning from data streams. All algorithms evaluated in this paper were implemented in the Java programming language by extending the MOA software.

We use the experimental framework for concept drift presented in [4]. Considering data streams as data generated from pure distributions, we can model 
a concept drift event as a weighted combination of two pure distributions that characterizes the target concepts before and after the drift. This framework defines the probability that a new instance of the stream belongs to the new concept after the drift based on the sigmoid function.

Definition 1. Given two data streams $a$, $b$, we define $c=a \oplus_{t_{0}}^{W} b$ as the data stream built by joining the two data streams $a$ and $b$, where $t_{0}$ is the point of change, $W$ is the length of change, $\operatorname{Pr}[c(t)=b(t)]=1 /\left(1+\mathrm{e}^{-4\left(t-t_{0}\right) / W}\right)$ and $\operatorname{Pr}[c(t)=a(t)]=1-\operatorname{Pr}[c(t)=b(t)]$.

In order to create a data stream with multiple concept changes, we can build new data streams joining different concept drifts, i. e. $\left(\left(\left(a \oplus_{t_{0}}^{W_{0}} b\right) \oplus_{t_{1}}^{W_{1}} c\right) \oplus_{t_{2}}^{W_{2}} d\right) \ldots$

\subsection{Datasets for concept drift}

Synthetic data has several advantages - it is easier to reproduce and there is little cost in terms of storage and transmission. For this paper we use the data generators most commonly found in the literature.

SEA Concepts Generator This artificial dataset contains abrupt concept drift, first introduced in [23]. It is generated using three attributes, where only the two first attributes are relevant. All the attributes have values between 0 and 10 . The points of the dataset are divided into 4 blocks with different concepts. In each block, the classification is done using $f_{1}+f_{2} \leq \theta$, where $f_{1}$ and $f_{2}$ represent the first two attributes and $\theta$ is a threshold value. The most frequent values are $9,8,7$ and 9.5 for the data blocks. In our framework, SEA concepts are defined as follows:

$$
\left(\left(\left(S E A_{9} \oplus_{t_{0}}^{W} S E A_{8}\right) \oplus_{2 t_{0}}^{W} S E A_{7}\right) \oplus_{3 t_{0}}^{W} S E A_{9.5}\right)
$$

Rotating Hyperplane This data was used as a testbed for CVFDT versus VFDT in [14]. Examples for which $\sum_{i=1}^{d} w_{i} x_{i} \geq w_{0}$ are labeled positive, and examples for which $\sum_{i=1}^{d} w_{i} x_{i}<w_{0}$ are labeled negative. Hyperplanes are useful for simulating time-changing concepts, because we can change the orientation and position of the hyperplane in a smooth manner by changing the relative size of the weights.

Random RBF Generator This generator was devised to offer an alternate complex concept type that is not straightforward to approximate with a decision tree model. The RBF (Radial Basis Function) generator works as follows: A fixed number of random centroids are generated. Each center has a random position, a single standard deviation, class label and weight. New examples are generated by selecting a center at random, taking weights into consideration so that centers with higher weight are more likely to be chosen. A random direction is chosen to offset the attribute values from the central point. Drift is introduced by moving the centroids with constant speed. 
LED Generator This data source originates from the CART book [5]. An implementation in $\mathrm{C}$ was donated to the UCI [1] machine learning repository by David Aha. The goal is to predict the digit displayed on a seven-segment LED display, where each attribute has a $10 \%$ chance of being inverted. The particular configuration of the generator used for the experiments (led) produces 24 binary attributes, 17 of which are irrelevant.

\subsection{Real-World Data}

The UCI machine learning repository [1] contains some real-world benchmark data for evaluating machine learning techniques. We consider three of the largest: Forest Covertype, Poker-Hand, and Electricity.

Forest Covertype Contains the forest cover type for $30 \times 30$ meter cells obtained from US Forest Service (USFS) Region 2 Resource Information System (RIS) data. It contains 581, 012 instances and 54 attributes, and it has been used in several papers on data stream classification $[10,20]$.

Poker-Hand Consists of 1, 000, 000 instances and 11 attributes. Each record of the Poker-Hand dataset is an example of a hand consisting of five playing cards drawn from a standard deck of 52. Each card is described using two attributes (suit and rank), for a total of 10 predictive attributes. There is one class attribute that describes the "Poker Hand".

Electricity is another widely used dataset described by M. Harries [11] and analysed by Gama [9]. This data was collected from the Australian New South Wales Electricity Market. In this market, prices are not fixed and are affected by demand and supply of the market. They are set every five minutes. The ELEC dataset contains 45, 312 instances. The class label identifies the change of the price relative to a moving average of the last 24 hours.

We use normalized versions of these datasets, so that the numerical values are between 0 and 1 . With the Poker-Hand dataset, the cards are not ordered, i.e. a hand can be represented by any permutation, which makes it very hard for propositional learners, especially for linear ones. We use a modified version, where cards are sorted by rank and suit, and have removed duplicates. This dataset loses about 171,799 examples, and comes down to 829, 201 examples.

These datasets are small compared to synthetic datasets we consider. Another important fact is that we do not know when drift occurs or indeed if there is any drift. We may simulate concept drift, joining the three datasets, merging attributes, and supposing that each dataset corresponds to a different concept

$$
\text { CovPokElec }=\left(\text { CoverType } \oplus_{581,012}^{5,000} \text { Poker }\right) \oplus_{1,000,000}^{5,000} \text { ELEC }
$$

As all examples need to have the same number of attributes, we simply concatenate all the attributes, and set the number of classes to the maximum number of classes of all the datasets. 

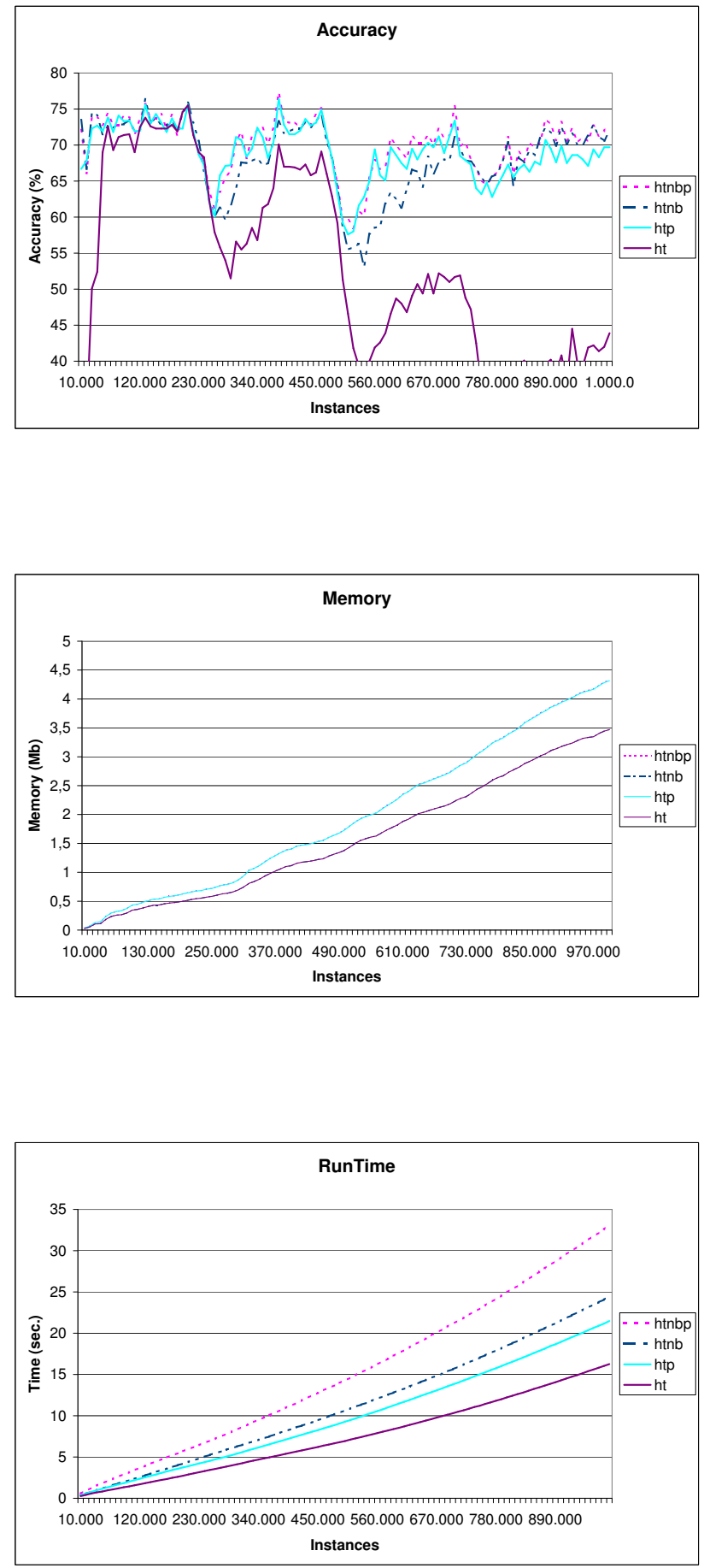

Fig. 2. Accuracy, runtime and memory on the LED data with three concept drifts. 


\begin{tabular}{|c|c|c|c|c|c|c|c|c|c|}
\hline & \multicolumn{3}{|c|}{ Perceptron } & \multicolumn{3}{|c|}{ Naive Bayes } & \multicolumn{3}{|c|}{ Hoeffding Tree } \\
\hline & Time & Acc. & Mem. & Time & Acc. & Mem. & Time & Acc. & Mem. \\
\hline $\operatorname{RBF}(0,0)$ & 1.83 & $72.69 \pm 0.28$ & 0.01 & 4.15 & $72.04 \pm 0.06$ & 0.01 & 5.84 & $\mathbf{8 6 . 7 5} \pm 0.83$ & 1.56 \\
\hline $\operatorname{RBF}(50,0.001)$ & 2.67 & $65.33 \pm 0.04$ & 0.01 & 5.02 & $53.23 \pm 0.05$ & 0.01 & 6.40 & $52.52 \pm 0.17$ & 1.50 \\
\hline $\operatorname{RBF}(10,0.001)$ & 1.82 & $74.11 \pm 0.09$ & 0.01 & 4.14 & $75.79 \pm 0.06$ & 0.01 & 5.80 & $83.72 \pm 0.58$ & 1.55 \\
\hline $\operatorname{RBF}(50,0.0001)$ & 2.64 & $69.34 \pm 0.07$ & 0.01 & 4.99 & $53.82 \pm 0.04$ & 0.01 & 7.04 & $55.78 \pm 0.33$ & 1.86 \\
\hline $\operatorname{RBF}(10,0.0001)$ & 1.85 & $73.92 \pm 0.16$ & 0.01 & 4.14 & $75.18 \pm 0.09$ & 0.01 & 5.94 & $83.59 \pm 0.50$ & 1.62 \\
\hline $\operatorname{HYP}(10,0.001)$ & 1.55 & $\mathbf{9 2 . 8 7} \pm 0.43$ & 0.01 & 3.91 & $77.64 \pm 3.74$ & 0.01 & 5.37 & $73.19 \pm 2.60$ & 1.60 \\
\hline $\operatorname{HYP}(10,0.0001)$ & 1.56 & $\mathbf{9 3 . 7 0} \pm 0.03$ & 0.01 & 3.90 & $90.23 \pm 0.76$ & 0.01 & 4.99 & $76.87 \pm 1.46$ & 1.40 \\
\hline $\operatorname{SEA}(50)$ & 1.17 & $87.15 \pm 0.05$ & 0.00 & 1.54 & $85.37 \pm 0.00$ & 0.00 & 2.54 & $85.68 \pm 0.00$ & 0.56 \\
\hline SEA $(50000)$ & 1.31 & $86.85 \pm 0.04$ & 0.00 & 1.69 & $85.38 \pm 0.00$ & 0.00 & 2.71 & $85.59 \pm 0.04$ & 0.56 \\
\hline $\operatorname{LED}(50000)$ & 6.64 & $72.76 \pm 0.01$ & 0.02 & 8.97 & $54.02 \pm 0.00$ & 0.04 & 12.81 & $52.74 \pm 0.15$ & 4.98 \\
\hline CovType & 12.21 & 81.68 & 0.05 & 22.81 & 60.52 & 0.08 & 13.43 & 68.30 & 2.59 \\
\hline POKER & 5.36 & 3.34 & 0.01 & 9.25 & 59.55 & 0.02 & 5.46 & 73.62 & 1.11 \\
\hline ELECTRICITY & 0.53 & 79.07 & 0.01 & 0.55 & 73.36 & 0.01 & 0.86 & 75.35 & 0.12 \\
\hline \multirow[t]{2}{*}{ CovPokElec } & 20.87 & 13.69 & 0.06 & 56.52 & 24.34 & 0.11 & 42.82 & 72.63 & 10.03 \\
\hline & \multicolumn{3}{|c|}{$\begin{array}{c}\text { 69.04 Acc. } \\
\text { 0.12 RAM-Hours }\end{array}$} & \multicolumn{3}{|c|}{$\begin{array}{c}67.18 \text { Acc. } \\
0.41 \text { RAM-Hours }\end{array}$} & \multicolumn{3}{|c|}{$\begin{array}{l}\mathbf{7 3 . 3 1} \text { Acc. } \\
\text { 37 RAM-Hours }\end{array}$} \\
\hline
\end{tabular}

Table 1. Comparison of Perceptron, Naive Bayes and Hoeffding Tree. The best individual accuracies are indicated in boldface.

\subsection{Results}

We use the datasets explained in the previous sections for evaluation. The experiments were performed on a $3 \mathrm{GHz}$ Intel 64-bit machine with $2 \mathrm{~GB}$ of memory. The evaluation methodology used was Interleaved Test-Then-Train on 10 runs: every example was used for testing the model before using it to train. This interleaved test followed by train procedure was carried out on 10 million examples from the hyperplane and RandomRBF datasets, and one million examples from the SEA dataset. The parameters of these streams are the following:

- $\operatorname{RBF}(x, v)$ : RandomRBF data stream with $x$ centroids moving at speed $v$.

- HYP $(x, v)$ : Hyperplane data stream with $x$ attributes changing at speed $v$.

- SEA $(v)$ : SEA dataset, with length of change $v$.

- LED $(v)$ : LED dataset, with length of change $v$.

Tables 1, 2 and 3 report the final accuracy, and speed of the classification models induced on the synthetic data and the real datasets: ForEsT CoverType, Poker Hand, Electricity and CovPokElec. Accuracy is measured as the final percentage of examples correctly classified over the test/train interleaved evaluation. Time is measured in seconds, and memory in MB. The classification methods used are the following: perceptron, naive Bayes, Hoeffding Naive Bayes Tree (hnbt), Hoeffding Perceptron Tree (hpt), Hoeffding Naive Bayes Perceptron Tree (hnbpt), and ADWIN bagging using hnbt, hpt, and hnbpt.

The learning curves and model growth curves for the LED dataset are plotted in Figure 2. We observe that ht and hpt are the fastest decision trees. As the trees do not need more space to compute naive Bayes predictions at the leaves, hnbt uses the same memory as ht, and hpnbt uses the same memory as hpt. On accuracy, ht is the method that adapts more slowly to change, and during some time intervals hpt performs better than hnbt, but in other intervals performs worse. hnbpt is always the most or very close to the most accurate method as 


\begin{tabular}{|c|c|c|c|c|c|c|c|c|c|}
\hline & \multicolumn{3}{|c|}{ hnbt } & \multicolumn{3}{|c|}{ hpt } & \multicolumn{3}{|c|}{ hnbpt } \\
\hline & Time & Acc. & Mem. & Time & Acc. & Mem. & Time & Acc. & Mem. \\
\hline $\operatorname{RBF}(0,0)$ & 9.03 & $90.78 \pm 0.46$ & 1.57 & 8.04 & $90.33 \pm 0.49$ & 2.30 & 10.77 & $\mathbf{9 1 . 0 7} \pm 0.44$ & 2.30 \\
\hline $\operatorname{RBF}(50,0.001)$ & 10.98 & $57.70 \pm 0.22$ & 1.51 & 8.66 & $68.95 \pm 0.31$ & 2.20 & 11.32 & $68.65 \pm 0.32$ & 2.20 \\
\hline $\operatorname{RBF}(10,0.001)$ & 9.07 & $87.24 \pm 0.29$ & 1.56 & 8.03 & $87.61 \pm 0.36$ & 2.28 & 10.77 & $87.98 \pm 0.32$ & 2.28 \\
\hline $\operatorname{RBF}(50,0.0001)$ & 11.62 & $69.00 \pm 0.46$ & 1.86 & 9.54 & $\mathbf{7 9 . 9 1} \pm 0.42$ & 2.72 & 12.45 & $79.88 \pm 0.39$ & 2.72 \\
\hline $\operatorname{RBF}(10,0.0001)$ & 9.28 & $88.47 \pm 0.37$ & 1.63 & 8.20 & $89.37 \pm 0.32$ & 2.38 & 10.98 & $\mathbf{8 9 . 7 4} \pm 0.33$ & 2.39 \\
\hline $\operatorname{HYP}(10,0.001)$ & 9.73 & $83.24 \pm 2.29$ & 1.61 & 7.57 & $82.74 \pm 1.13$ & 2.34 & 11.45 & $84.54 \pm 1.40$ & 2.34 \\
\hline $\operatorname{HYP}(10,0.0001)$ & 9.37 & $\mathbf{8 8 . 4 2} \pm 0.36$ & 1.40 & 7.08 & $82.59 \pm 0.62$ & 2.04 & 11.30 & $88.40 \pm 0.36$ & 2.04 \\
\hline $\operatorname{SEA}(50)$ & 3.70 & $86.63 \pm 0.00$ & 0.57 & 4.65 & $86.73 \pm 0.01$ & 1.23 & 5.49 & $\mathbf{8 7 . 4 1} \pm 0.01$ & 1.24 \\
\hline SEA $(50000)$ & 4.51 & $86.44 \pm 0.03$ & 0.57 & 4.85 & $86.41 \pm 0.07$ & 1.23 & 5.64 & $87.12 \pm 0.07$ & 1.24 \\
\hline $\operatorname{LED}(50000)$ & 21.28 & $68.06 \pm 0.10$ & 4.99 & 18.64 & $68.87 \pm 0.07$ & 6.00 & 24.99 & $\mathbf{7 0 . 0 4} \pm 0.03$ & 6.00 \\
\hline CovType & 24.73 & 81.06 & 2.59 & 16.53 & 83.59 & 3.46 & 22.16 & 85.77 & 3.46 \\
\hline POKER & 9.81 & 83.05 & 1.12 & 8.40 & 74.02 & 1.82 & 11.40 & 82.93 & 1.82 \\
\hline ELECTRICITY & 0.96 & 80.69 & 0.12 & 0.93 & 84.24 & 0.21 & 1.07 & 84.34 & 0.21 \\
\hline \multirow[t]{2}{*}{ COVPokElec } & 68.37 & 83.41 & 10.05 & 49.37 & 73.33 & 13.53 & 69.70 & 83.28 & 13.53 \\
\hline & \multicolumn{3}{|c|}{$\begin{array}{c}\text { 81.01 Acc. } \\
\text { 61.58 RAM-Hours }\end{array}$} & \multicolumn{3}{|c|}{$\begin{array}{c}\text { 81.33 Acc. } \\
\text { 68.56 RAM-Hours }\end{array}$} & \multicolumn{3}{|c|}{$\begin{array}{c}\mathbf{8 3 . 6 5} \text { Acc. } \\
\text { 93.84 RAM-Hours }\end{array}$} \\
\hline
\end{tabular}

Table 2. Comparison of hnbt, hpt, and hnbpt algorithms. The best individual accuracies are indicated in boldface.

it is capable of making use of the decision of the majority class, naive Bayes and perceptron.

Table 1 shows the accuracy, speed and memory usage of a naive Bayes learner, a perceptron with $\eta=1$ and a classic Hoeffding Tree with majority class learning at the leaves. As naive Bayes uses a Gaussian distribution to model numeric attributes, with different variances for each class, it does not yield a linear separator as the perceptron does. In general terms, we see that the perceptron and the Hoeffding Tree are the fastest methods, but the Hoeffding Tree needs more memory. Comparing using RAM-Hours, naive Bayes needs 3.5 times more RAMHours than the perceptron, and the Hoeffding Tree needs 89 more RAM-Hours than naive Bayes. Note that using $\eta=1$ we obtain a very fast adaptive method, but for some datasets like POKER, the results are worse than obtained using a more conservative rate like $\eta=0.01$. Choosing an optimal $\eta$ remains an open problem for further research.

Table 2 shows, for the Hoeffding tree models, their accuracy, speed and memory. We see that hpt is much faster than hnbt, and more accurate in several streams. hnbpt is more accurate than hnbt and hpt, but it needs more time. Comparing RAM-Hours, hpt needs 1.11 times more RAM-Hours than hnbt, and 2.54 more RAM-Hours than ht, and hnbpt needs 1.37 more than hpt.

Table 3 reports the accuracy, speed and memory of ADWIN bagging using hnbt, hpt, and hnbpt. ADWIN bagging using hnbpt is the most accurate method, but it uses more time and memory than the other variants. In RAM-Hours, it needs 1.62 times more than ADWIN bagging using $\mathbf{h p t}$, and 1.51 times more than ADWIN bagging using hnbt. ADWIN bagging using hpt needs fewer resources than ADWIN bagging using hnbt.

Comparing hnbpt from Table 2 with the single perceptron from Table 1, we obtain a $20 \%$ better accuracy, but at a cost of 780 times the amount of RAM- 


\begin{tabular}{|c|c|c|c|c|c|c|c|c|c|}
\hline & \multicolumn{3}{|c|}{ ADWIN Bagging hnbt } & \multicolumn{3}{|c|}{ ADWIN Bagging hpt } & \multicolumn{3}{|c|}{ ADWIN Bagging hnbpt } \\
\hline & Time & Acc. & Mem & Time & Acc. & Mem & Time & Acc. & Mem \\
\hline $\operatorname{RBF}(0,0)$ & 102.22 & $94.30 \pm 0.07$ & 16.22 & 88.84 & $93.70 \pm 0.10$ & 23.35 & 115.31 & $\mathbf{9 4 . 3 6} \pm 0.07$ & 23.98 \\
\hline $\operatorname{RBF}(50,0.001)$ & 88.75 & $67.67 \pm 0.16$ & 0.12 & 41.15 & $\mathbf{7 4 . 8 2} \pm 0.26$ & 2.27 & 76.34 & $74.13 \pm 0.40$ & 2.84 \\
\hline $\operatorname{RBF}(10,0.001)$ & 97.65 & $89.74 \pm 0.09$ & 12.97 & 80.65 & $90.36 \pm 0.11$ & 20.44 & 106.64 & $\mathbf{9 0 . 5 5} \pm 0.10$ & 19.73 \\
\hline $\operatorname{RBF}(50,0.0001)$ & 90.64 & $84.99 \pm 0.17$ & 1.33 & 61.82 & $87.24 \pm 0.14$ & 9.66 & 94.22 & $87.97 \pm 0.11$ & 9.39 \\
\hline $\operatorname{RBF}(10,0.0001)$ & 100.21 & $91.97 \pm 0.07$ & 13.81 & 82.54 & $92.53 \pm 0.12$ & 21.56 & 109.59 & $\mathbf{9 3 . 0 1} \pm 0.05$ & 21.32 \\
\hline $\operatorname{HYP}(10,0.001)$ & 90.77 & $89.92 \pm 0.31$ & 3.02 & 31.86 & $91.20 \pm 0.99$ & 1.11 & 69.23 & $\mathbf{9 1 . 4 5} \pm 0.81$ & 2.43 \\
\hline $\operatorname{HYP}(10,0.0001)$ & 107.36 & $91.30 \pm 0.21$ & 8.22 & 30.77 & $93.63 \pm 0.21$ & 0.08 & 50.57 & $93.61 \pm 0.24$ & 0.11 \\
\hline $\operatorname{SEA}(50)$ & 44.48 & $88.22 \pm 0.22$ & 4.33 & 54.09 & $88.08 \pm 0.07$ & 11.07 & 59.19 & $\mathbf{8 8 . 6 0} \pm 0.09$ & 10.17 \\
\hline SEA(50000) & 41.03 & $88.61 \pm 0.07$ & 2.69 & 53.44 & $87.85 \pm 0.07$ & 10.78 & 54.07 & $88.65 \pm 0.05$ & 6.83 \\
\hline $\operatorname{LED}(50000)$ & 150.62 & $\mathbf{7 3 . 1 4} \pm 0.02$ & 5.09 & 93.09 & $72.82 \pm 0.03$ & 14.84 & 130.56 & $73.02 \pm 0.02$ & 8.45 \\
\hline CovTyPe & 165.75 & 85.73 & 0.80 & 50.06 & 86.33 & 1.66 & 115.58 & 87.88 & 1.25 \\
\hline POKER & 57.40 & 74.56 & 0.09 & 37.14 & 65.76 & 0.21 & 73.41 & 74.36 & 0.16 \\
\hline ELECTRICITY & 3.17 & 84.36 & 0.13 & 2.59 & 85.22 & 0.44 & 3.55 & 86.44 & 0.30 \\
\hline \multirow[t]{2}{*}{ CovPoKElec } & 363.70 & 78.96 & 1.18 & 118.64 & 67.02 & 1.13 & 402.20 & 78.77 & 1.54 \\
\hline & \multicolumn{3}{|c|}{$\begin{array}{c}84.53 \text { Acc. } \\
\text { 1028.02 RAM-Hours }\end{array}$} & \multicolumn{3}{|c|}{$\begin{array}{c}\text { 84.04 Acc. } \\
\text { 957.38 RAM-Hours }\end{array}$} & \multicolumn{3}{|c|}{$\begin{array}{c}\mathbf{8 5 . 9 1} \text { Acc. } \\
\text { 1547.33 RAM-Hours }\end{array}$} \\
\hline
\end{tabular}

Table 3. Comparison of ADWIN bagging with hnbt, hpt, and hnbpt. The best individual accuracies are indicated in boldface.

Hours. Comparing ADWIN bagging using hnbpt (Table 3) with a single hnbpt (Table 2) we obtain a $3 \%$ better accuracy, at 16.50 times the RAM-Hours.

Concept drift is handled well by the proposed ADWIN bagging algorithms, excluding the poor performance of the hpt-based classifier on CovPoKELEC, which is due to the nature of the POKER dataset. Decision trees alone do not deal as well with evolving streaming data, as they have limited capability of adaption.

A tradeoff between RAM-Hours and accuracy could be to use single perceptrons when resources are scarce, and ADWIN bagging methods when more accuracy is needed. Note that to gain an increase of $24 \%$ in accuracy, we have to increase by more than 10,000 times the RAM-Hours needed; this is the difference of magnitude between the RAM-Hours needed for a single perceptron and for a more accurate ADWIN bagging method.

\section{Conclusions}

We have investigated four perceptron-based methods for data streams: a single learner, a decision tree, a hybrid tree, and an ensemble method. These methods use perceptrons with a sigmoid activation function, optimizing the squared error, with one perceptron per class value. We observe that perceptron-based methods are competitive in accuracy and use of resources. We have introduced the use of RAM-Hours as a performance measure. Using RAM-Hours, it is easy to compare resources used by classifier algorithms.

As future work, we would like to build new methods based on the perceptron, with an adaptive learning rate. We think that in changing scenarios, using a flexible learning rate may allow us to obtain more accurate methods, without incurring large additional runtime or memory costs. 


\section{References}

1. A. Asuncion and D. Newman. UCI machine learning repository, 2007.

2. K. Bennett, N. Cristianini, J. Shawe-Taylor, and D. Wu. Enlarging the margins in perceptron decision trees. Machine Learning, 41(3):295-313, 2000.

3. A. Bifet and R. Gavaldà. Learning from time-changing data with adaptive windowing. In $S D M, 2007$.

4. A. Bifet, G. Holmes, B. Pfahringer, R. Kirkby, and R. Gavaldà. New ensemble methods for evolving data streams. In $K D D$, pages 139-148, 2009.

5. L. Breiman, J. H. Friedman, R. A. Olshen, and C. J. Stone. Classification and Regression Trees. Wadsworth, 1984.

6. P. Domingos and G. Hulten. Mining high-speed data streams. In $K D D$, pages $71-80,2000$

7. E. Frank, Y. Wang, S. Inglis, G. Holmes, and I. H. Witten. Using model trees for classification. Machine Learning, 32(1):63-76, 1998.

8. J. Gama. On Combining Classification Algorithms. VDM Verlag, 2009.

9. J. Gama, P. Medas, G. Castillo, and P. P. Rodrigues. Learning with drift detection. In $S B I A$, pages 286-295, 2004.

10. J. Gama, R. Rocha, and P. Medas. Accurate decision trees for mining high-speed data streams. In KDD, pages 523-528, 2003.

11. M. Harries. Splice-2 comparative evaluation: Electricity pricing. Technical report, The University of South Wales, 1999.

12. G. Holmes, R. Kirkby, and B. Pfahringer. Stress-testing Hoeffding trees. In PKDD, pages 495-502, 2005.

13. G. Holmes, R. Kirkby, and B. Pfahringer. MOA: Massive Online Analysis. http://sourceforge.net/projects/moa-datastream. 2007.

14. G. Hulten, L. Spencer, and P. Domingos. Mining time-changing data streams. In $K D D$, pages $97-106,2001$.

15. E. Ikonomovska and J. Gama. Learning model trees from data streams. In Discovery Science, pages 52-63, 2008.

16. E. Ikonomovska, J. Gama, R. Sebastião, and D. Gjorgjevik. Regression trees from data streams with drift detection. In Discovery Science, pages 121-135, 2009.

17. N. Landwehr, M. Hall, and E. Frank. Logistic model trees. Machine Learning, 59(1-2):161-205, 2005.

18. S. K. Murthy. Automatic construction of decision trees from data: A multidisciplinary survey. Data Min. Knowl. Discov., 2(4):345-389, 1998.

19. N. Oza and S. Russell. Online bagging and boosting. In Artificial Intelligence and Statistics 2001, pages 105-112. Morgan Kaufmann, 2001.

20. N. C. Oza and S. J. Russell. Experimental comparisons of online and batch versions of bagging and boosting. In KDD, pages 359-364, 2001.

21. S. R. Safavian and D. Landgrebe. A survey of decision tree classifier methodology. Systems, Man and Cybernetics, IEEE Transactions on, 21(3):660-674, 1991.

22. J. C. Schlimmer and D. H. Fisher. A case study of incremental concept induction. In $A A A I$, pages $496-501,1986$.

23. W. N. Street and Y. Kim. A streaming ensemble algorithm (SEA) for large-scale classification. In KDD, pages 377-382, 2001.

24. P. E. Utgoff. Perceptron trees: A case study in hybrid concept representations. In $A A A I$, pages $601-606,1988$.

25. T. Velte, A. Velte, and R. Elsenpeter. Cloud Computing, A Practical Approach. McGraw-Hill, Inc., New York, NY, USA, 2010.

26. Z. Zhou and Z. Chen. Hybrid decision tree. Knowledge-based systems, 15(8):515$528,2002$. 